\title{
Parallax measurements of cool brown dwarfs ${ }^{\star}, \star \star$
}

\author{
E. Manjavacas ${ }^{1}$, B. Goldman ${ }^{1}$, S. Reffert ${ }^{2}$, and T. Henning ${ }^{1}$ \\ 1 Max Planck Institute für Astronomie, Königstuhl 17, 69117 Heidelberg, Germany \\ e-mail: [manjavacas;goldman; henning]@mpia.de \\ 2 University of Heidelberg, Zentrum für Astronomie, Landessternwarte Königstuhl 12, 69117 Heidelberg, Germany \\ e-mail: sreffert@lsw.uni-heidelberg.de
}

Received 17 April 2013 / Accepted 3 October 2013

\begin{abstract}
Context. Accurate parallax measurements allow us to determine physical properties of brown dwarfs and help us constrain evolutionary and atmospheric models, break age-mass degeneracy, and reveal unresolved binaries.

Aims. We measured absolute trigonometric parallaxes and proper motions of six cool brown dwarfs using background galaxies to establish an absolute reference frame. We derive the absolute $J$-band magnitude. The six T brown dwarfs in our sample have spectral types between T2.5 and T8 and magnitudes between 13.9 and 18.0 in the Two Micron All Sky Survey (2MASS) with photometric distances below $25 \mathrm{pc}$.

Methods. The observations were taken in the $J$-band with the Omega-2000 camera on the $3.5 \mathrm{~m}$ telescope at Calar Alto during a time period of 27 months between March 2011 and June 2013. The number of epochs varied between 11 and 12 depending on the object. The reduction of the astrometric measurements was carried out with respect to the field stars. The relative parallax and proper motions were transformed into absolute measurements using the background galaxies in our fields.

Results. We obtained absolute parallaxes for our six brown dwarfs with a precision between 3 and 6 mas. We compared our results in a color-magnitude diagram with other brown dwarfs with determined parallax and with the BT-Settl 2012 atmospheric models. For four of the six targets, we found a good agreement in luminosity with objects of similar spectral types. We obtained an improved accuracy in the parallaxes and proper motions in comparison to previous works. The object 2MASS J11061197+2754225 is more than 1 mag overluminous in all bands, which point to binarity or high order multiplicity.
\end{abstract}

Key words. stars: low-mass - brown dwarfs - infrared: stars - astrometry - parallaxes - proper motions

\section{Introduction}

Since the discovery of the first brown dwarfs (BDs; GD165 by Becklin \& Zuckerman 1988; PPl 15 by Basri et al. 1995; Teide 1 by Rebolo et al. 1995 and Gliese229B by Nakajima et al. 1995), more than $1000 \mathrm{~L}$ and $\mathrm{T}$ type brown dwarfs have been discovered $^{1}$.

During their evolution BDs cool and dim. During their life, they change their spectral type because the effective temperature $\left(T_{\text {eff }}\right)$ decreases with the exception of BDs in the $\mathrm{L} / \mathrm{T}$ transition in which the $T_{\text {eff }}$ is roughly constant. For a given BD with a given temperature, the interval of masses and ages that the BD could have is very wide, so that the age and the mass are degenerate. The $T_{\text {eff }}$ is the physical parameter that drives the major changes in the observable photometric and spectroscopic features of brown dwarfs. However, among the known brown dwarfs emerge outliers, which show that secondary parameters are also responsible for brown dwarf properties, such as gravity, metallicity, and cloud variability (Burrows et al. 2006; Burgasser et al. 2006a; Liu et al. 2007).

\footnotetext{
* Based on observations taken with Omega-2000 at the $3.5 \mathrm{~m}$ telescope at the Centro Astronómico Hispano Alemán (CAHA) at Calar Alto, operated by the Max Planck Institut für Astronomie and the Instituto de Astrofísica de Andalucía (CSIC).

$\star \star$ Appendix $\mathrm{A}$ is available in electronic form at

http://www . aanda.org

1 www.dwarfarchives.org
}

Accurate measurements of distances allow us to determine physical properties of these objects, including luminosities or absolute fluxes to check atmospheric models, temperatures, space motions, and space densities.

Since our goal is to derive parallaxes with a high accuracy (5-10\%), we constrained our sample to objects with photometric distances up to $25 \mathrm{pc}$. Our sample of objects is located at spectrophotometric distances of $10-25 \mathrm{pc}$ with magnitudes in $J$ between 13.9 and 18.0, which are suitable for astrometry.

Since the first parallax programs for brown dwarfs began with Dahn et al. (2002), Vrba et al. (2004), and Tinney et al. (2003), the relationship between the color and magnitude of BDs has been studied (Burgasser et al. 2008; Schilbach et al. 2009; Marocco et al. 2010; Faherty et al. 2012; Dupuy \& Liu 2012) among others. One of the most significant results of these studies is the large dispersion in luminosity for objects with similar spectral types (Faherty et al. 2012), which shows the importance of other factors, such as gravity, metallicity, sedimentation, and binarity (Tsuji et al. 1996; Burrows et al. 2006; Saumon \& Marley 2008). Increasing the number of cool brown dwarfs with accurate distance measurements allows us to understand the variation in the color magnitude and H-R diagrams, as we can determine the luminosity more accurately. Also, the $J$-band bump in the color-magnitude diagram, a brightening observed in the $J$ band for brown dwarfs with spectral types between T1 and T5, is not well understood (Burgasser et al. 2002b; Tinney et al. 2003 and Vrba et al. 2004). There are still few objects with parallaxes 
Table 1. Sample of objects with their spectral types, photometry and references for our targets.

\begin{tabular}{lcccccccc}
\hline \hline Name & SpT & $J[\mathrm{mag}]$ & $H[\mathrm{mag}]$ & $K_{\mathrm{s}}[\mathrm{mag}]$ & Ref. objects & exS & Epochs & Reference \\
\hline 2MASS J11061197+2754225 & $\mathrm{T} 2.5(1)$ & $14.96 \pm 0.04$ & $14.20 \pm 0.05$ & $13.84 \pm 0.05$ & 96 & 49 & 10 & $(1)$ \\
ULAS J130217.21+130851.2 & T8 (5) & $18.11 \pm 0.04$ & $18.60 \pm 0.06$ & $18.28 \pm 0.03$ & 247 & 68 & 12 & $(2)$ \\
ULAS J141756.22+133045.8 & T5.5(3) & $16.77 \pm 0.01$ & $17.00 \pm 0.03$ & $17.00 \pm 0.04$ & 77 & 99 & 10 & $(3)$ \\
2MASS J22541892+3123498 & T4 (4) & $15.32 \pm 0.05$ & $15.06 \pm 0.08$ & $14.99 \pm 0.15$ & 644 & 125 & 10 & $(4)$ \\
ULAS J232035.28+144829.8 & T6 (6) & $16.79 \pm 0.02$ & $17.14 \pm 0.04$ & $17.40 \pm 0.03$ & 298 & 46 & 12 & $(3)$ \\
ULAS J232123.79+135454.9 & T7.5(2) & $16.69 \pm 0.03$ & $17.09 \pm 0.06$ & $17.36 \pm 0.10$ & 278 & 73 & 11 & $(3)$ \\
\hline
\end{tabular}

References. (1) Looper et al. (2007); (2) Burningham et al. (2010); (3) Scholz (2010); (4) Burgasser et al. (2002a); (5) Cushing et al. (2011); (6) Murray et al. (2011).

in the $\mathrm{L} / \mathrm{T}$ transition, which prevent the progress of understanding brown dwarf evolution.

In this paper, we report the absolute parallaxes and the absolute proper motions of six T ultra cool dwarfs with spectral types between T2.5 and T8 and spectrophotometric distances between $10-25 \mathrm{pc}$.

In Sect. 2, we explain how our selection criteria are used to select our targets and how the observations were carried out. In Sect. 3, we describe the data reduction: how the astrometry was performed, the estimation of the differential chromatic refraction (DCR), and the calculation of the relative and absolute parallax and proper motions. In Sect. 4, we compare our results to those of other previous studies, which included our object. Finally, we present our conclusions in Sect. 6.

\section{Observations}

Initially, we selected eigth $\mathrm{T}$ dwarfs with expected spectrophotometric distances smaller than $25 \mathrm{pc}$. Spectrophotometric distances of our targets were estimated using the relation given by Goldman et al. (2010). Only objects brighter than 18 mag in the $J$ band were selected, so that good signal-to-noise (S/N) observations could be obtained in a reasonable amount of time. Two targets were discarded later from the list, since they were located in fields with bright stars nearby, which compromised the accuracy of the astrometry. Finally, all the targets had to be observable most part of the year to have a better coverage of the parallax ellipse. In Table 1, we present an overview of our targets, which provide spectral types and derived JHK photometry in the MKO (Mauna Kea Observatories) system (Stephens \& Leggett 2004) for targets with 2MASS photometry. For the rest, we provide UKIDSS (UKIRT Infrared Deep Sky Survey) photometry, which is similar to MKO (Hewett et al. 2006). For the target ULAS J232035.28+144829.8, we provide the photometry from Murray et al. (2011), which is also similar to MKO. In the last column, we add references to the discovery papers.

Images were taken with the near-IR camera Omega-2000 on the $3.5 \mathrm{~m}$ telescope at Calar Alto in $J$ band. Omega-2000 is a prime-focus, near-IR camera with a wide field that uses a $2 \mathrm{k} \times 2 \mathrm{k}$ focal plane array with sensitivity from the $z$ band to the $K$ band. The camera provides a $15.4^{\prime} \times 15.4^{\prime}$ field of view with a resolution of $0.45^{\prime \prime} /$ pixel. The wide field of Omega-2000 allows us to convert from relative parallaxes and proper motions to absolute values using galaxies (between 50 and 130 galaxies in each field). The astrometric observations were taken in service mode in the $J$ band. In all the cases, 15 single frames with exposure times of $60 \mathrm{~s}$ were taken with dithering.

Our observations have been taken between March 2011 and June 2013 in 24 epochs. The baseline varies from 23 to 27 months. In the case of the object ULAS
J232035.28 + 144829.8, we also used one observation, which was taken in July 2009 with Omega-2000 in the methane filter; therefore, the baseline for this object is almost four years. The average seeing on Calar Alto during our observations was around $1.0^{\prime \prime}$. We typically observed at $1-2 \mathrm{~h}$ from the meridian, so that DCR might be significant (Stone 1996; Pravdo \& Shaklan 1996). Further details about the estimation of the DCR corrections are given in Sect. 3.2.

Dark frames and sky flats were taken every evening, and the bad pixel mask was derived from the dark current analysis by an appropriate cut in the goodness-of-fit of the linear relation between dark current and exposure time. The fifteen individual raw images were corrected using flats, darks and bad pixels mask frames. To perform the reduction of the raw images, we used the MPIA Omega-2000 pipeline, which runs under MIDAS. The outputs are single, calibrated images for each epoch. Before performing the analysis of our images, we stacked the fifteen single exposures to get a better S/N. The stacking process was carried out using the algorithm explained in Mutchler \& Fruchter (1997) and Fruchter \& Hook (2002). The single images are slightly shifted, so they must be previously aligned. These images are aligned in a world coordinate system, so that corresponding astronomical objects are stacked on top of each other. A median image of the shifted frames is extracted. Since the images were shifted with different offsets, only the central area overlaps for all frames. The outer regions, where individual images do not contribute are marked and left blank. To check if the stacking actually improved the final value for the parallax and proper motions, we repeated the analysis using the fifteen single frames and the result obtained was less accurate than using stacked images. Therefore, we used the stacked frames for the final analysis.

\section{Analysis}

\subsection{Astrometry}

We obtained positional measurements for all of the sources in each field from $S$ Extractor (Bertin \& Arnouts 1996) using the parameter XWIN_IMAGE.SExtractor determines the background and identifies whether pixels belong to background or to objects. The program splits up the area that is not background into separate objects and determines the properties of each object. The output from SExtractor is a catalog for each epoch and field that contains all the objects in each field, the positions with error, instrumental magnitudes and errors, instrumental fluxes and errors, and star/galaxy classification among other parameters. The errors in position provided by SExtractor are estimated using photon statistics. This estimate is considered to be a lower value of the real error. 
The next step to create an astrometric catalog with the objects in our fields was to associate the detections in the multiple epochs that belonged to a common set of objects. For that, we cross-identified stars and we matched detections in a given frame to an astrometric reference catalog. As the telescope did not provide WCS information in the image headers, we used the software from www . astrometry . net (Lang et al. 2010) to perform a preliminary astrometry. The reference catalog used is the USNO-B1.0 catalog (Monet et al. 2003). This preliminary astrometry cannot be better than the accuracy of the catalog, which is around 200 mas. Then, we refined the initial guess using the software SCAMP (Bertin 2006) by choosing the first stacked image of the first epoch for each target as reference catalog. The reference objects used to perform the astrometry were distributed uniformly in the fields. These fields contained between 70 and 650 references. A different weight was given to the high $\mathrm{S} / \mathrm{N}$ reference objects and the small $\mathrm{S} / \mathrm{N}$ reference objects.

We constructed the catalog of associated detections by starting with the list of detections in the first stacked image on the first epoch, and then adding detections from the next epoch by finding matches between the objects in the catalogs. We performed the match of the catalogs using the IRAF routine tables.ttools.tmatch. This routine deletes the objects, which are not detected in all the epochs, but mainly faint targets and stars at the edges of the fields. We used a search radius of $1^{\prime \prime}$ around the object from the reference catalog.

\subsection{DCR correction}

The DCR effect results in astrometric shifts of the centroids (in any single-band imaging survey) because of the dependence of the refractive index of air on the wavelength. The reference objects and the targets have a different flux distribution in the $J$ band because they have different spectral types. Thus, their positions shift relative to one another due to different amounts of atmospheric refraction. Therefore, the DCR is a potential source of astrometric error, and it must be estimated.

In our case, the observations were typically performed between 1 and $2 \mathrm{~h}$ from the meridian. To estimate the magnitude of the resulting DCR for the field stars and the targets, we used the formula according to Monet et al. (1992), Stone (1996), Pravdo \& Shaklan (1996), and Kaczmarczik et al. (2009). We calculated the DCR effect for the targets relative to the typical field stars (M dwarfs). The correction due to DCR was typically 1 mas for the relative position between target and field stars. Since our typical parallax errors are of the order of 3-6 mas (see Sect. 3.3.2), it was not necessary to take the DCR into account. We also checked the influence of an epoch taken far from the meridian in the final result for the parallax. The conclusion was that the final parallax value did not change significantly. We discuss other astrometric error sources in detail in Sect. 3.4.

\subsection{Parallaxes}

\subsubsection{Relative parallaxes}

For the target and each object in the field, we use the positions in each epoch with their errors as an input to a $\chi^{2}$ fit. Errors used for the fitting are the residuals in right ascension (RA) and declination (Dec), respectively, and have been determined iteratively. These errors include all the error sources explained in Sect. 3.4. We fit the positions $\left(\alpha_{0}\right.$ and $\left.\delta_{0}\right)$, proper motions $\left(\mu_{\alpha}\right.$ and $\left.\mu_{\delta}\right)$, and the parallax $(\pi)$. Each fit to $2 \times N_{\text {epochs }}$ measurements had $2 \times N_{\text {epochs }}-5$ degrees of freedom. It is important to note that this parallax is a relative parallax, which uses field objects in the field of the target as references.

The apparent trajectory of each object in the field was then fitted to an astrometric model:

$$
\Delta \alpha(t)=\mu_{\alpha}\left(t-t_{0}\right)+\pi \cdot\left(p_{\alpha}(t)-p_{\alpha}\left(t_{0}\right)\right)
$$$$
\Delta \delta(t)=\mu_{\delta}\left(t-t_{0}\right)+\pi \cdot\left(p_{\delta}(t)-p_{\delta}\left(t_{0}\right)\right),
$$

where $\Delta \alpha(t)$ and $\Delta \delta(t)$ are the positional offsets with respect to the first epoch of observation at $t_{0} ; t$ is the time; $\mu_{\alpha}$ and $\mu_{\delta}$ are the proper motion in RA and Dec, $\pi$ is the parallax; and $p_{\alpha}$ and $p_{\delta}$ are the parallax factors in RA and Dec, respectively.

The parallax factors were computed using the Earth geocenter as obtained from the JPL DE405 solar system ephemeris. This model is based on the methods in the HIPPARCos (Perryman 1997) and Tycho Catalogues (Hog et al. 2000).

We present the plots of the stellar paths obtained using Eqs. (1) and (2) in the Appendix.

\subsubsection{Correction from relative to absolute parallax}

As mentioned before, the parallaxes from the astrometric solution are relative to the position of the background objects chosen as references. In general, these objects were field objects that may have their own parallaxes and proper motions, so that a correction is based upon the true parallaxes of the reference objects to convert to an absolute measurement. The field objects used as references are weighted depending on their $S / N$, giving more weight to the objects with better $\mathrm{S} / \mathrm{N}$. Given the large field of view of Omega-2000 $\left(15.4^{\prime} \times 15.4^{\prime}\right)$, we can find a sufficient number of extragalactic sources in all our fields. To derive the absolute parallax from the relative parallax, we used the extragalactic sources (exS) that we found in the fields of our targets. We searched for the extragalactic sources by setting the keyword CLASS_STAR in SExtractor. This software classifies extragalactic sources and stars in a field using neural networks, as explained in Bertin \& Arnouts (1996) by applying a method called backpropagation. Afterward, we determined the relative parallaxes of all the objects that were classified as extragalactic sources. We made a histogram of parallaxes and proper motions of these sources. As we could detect plenty of outliers in the histograms, we removed the objects with parallaxes or proper motions that were further than 3- $\sigma$ away from the median in parallaxes or proper motions. We fitted a Gaussian function to the histogram of the parallaxes after deleting these outliers. Finally, the relative parallax for the extragalactic sources $\left(\pi_{\mathrm{exS}}\right)$ is the mean of the fitted Gaussian, and the error of this parallax is the error in the mean for the Gaussian fitted to our data.

We correct the relative parallax to the absolute parallax as follows: $\pi_{\mathrm{abs}}=\pi_{\mathrm{rel}}-\pi_{\mathrm{exS}}$, where $\pi_{\mathrm{abs}}$ is the absolute parallax for the object, $\pi_{\text {rel }}$ is the relative parallax of the object, and $\pi_{\text {exS }}$ is derived as described above.

We execute a similar procedure to calculate the absolute proper motions given in Table 2.

\subsection{Absolute parallax accuracy}

We have several error sources, which arise at different phases in our analysis:

- Centroid errors. These errors are calculated by SExtractor using an iterative method. The positional uncertainties due to image centroiding in the relative position of the target is on average $\sim 1.5$ mas. 
Table 2. Summary of the results.

\begin{tabular}{lccccccc}
\hline \hline Object & $\mu_{\alpha}(\mathrm{mas} / \mathrm{yr})$ & $\mu_{\delta}(\mathrm{mas} / \mathrm{yr})$ & $\pi_{\text {rel }}(\mathrm{mas})$ & $\pi_{\text {abs }}(\mathrm{mas})$ & $d(\mathrm{pc})$ & $d_{\text {phot }}(\mathrm{pc})$ & $\chi^{2} / N_{\text {d.o.f. }}$ \\
\hline 2M J1106+2754 & $-311 \pm 4$ & $-438 \pm 5$ & $46 \pm 3$ & $48 \pm 3$ & $20.6_{-1.2}^{+1.0}$ & $12.5 \pm 1.4$ & $21.8 / 17$ \\
ULAS J1302+1308 & $-445 \pm 6$ & $5 \pm 7$ & $67 \pm 5$ & $65 \pm 5$ & $15.4_{-1.4}^{+1.1}$ & $16.1 \pm 2.3$ & $25.4 / 19$ \\
ULAS J1417+1330 & $-121 \pm 4$ & $50 \pm 3$ & $32 \pm 3$ & $33 \pm 3$ & $30.3_{-3.8}^{+2.5}$ & $23.8 \pm 5.1$ & $23.0 / 17$ \\
2M J2254+3123 & $67 \pm 3$ & $187 \pm 7$ & $71 \pm 2$ & $72 \pm 3$ & $13.9_{-0.6}^{+0.5}$ & $14.7 \pm 1.9$ & $23.4 / 17$ \\
ULAS J2320+1448 & $410 \pm 4$ & $121 \pm 3$ & $47 \pm 3$ & $47 \pm 4$ & $21.1_{-2.2}^{+1.6}$ & $20.7 \pm 3.6$ & $22.6 / 19$ \\
ULAS J2321+1354 & $76 \pm 4$ & $-576 \pm 6$ & $83 \pm 3$ & $84 \pm 4$ & $11.8_{-0.6}^{+0.5}$ & $10.8 \pm 0.7$ & $22.2 / 19$ \\
\hline
\end{tabular}
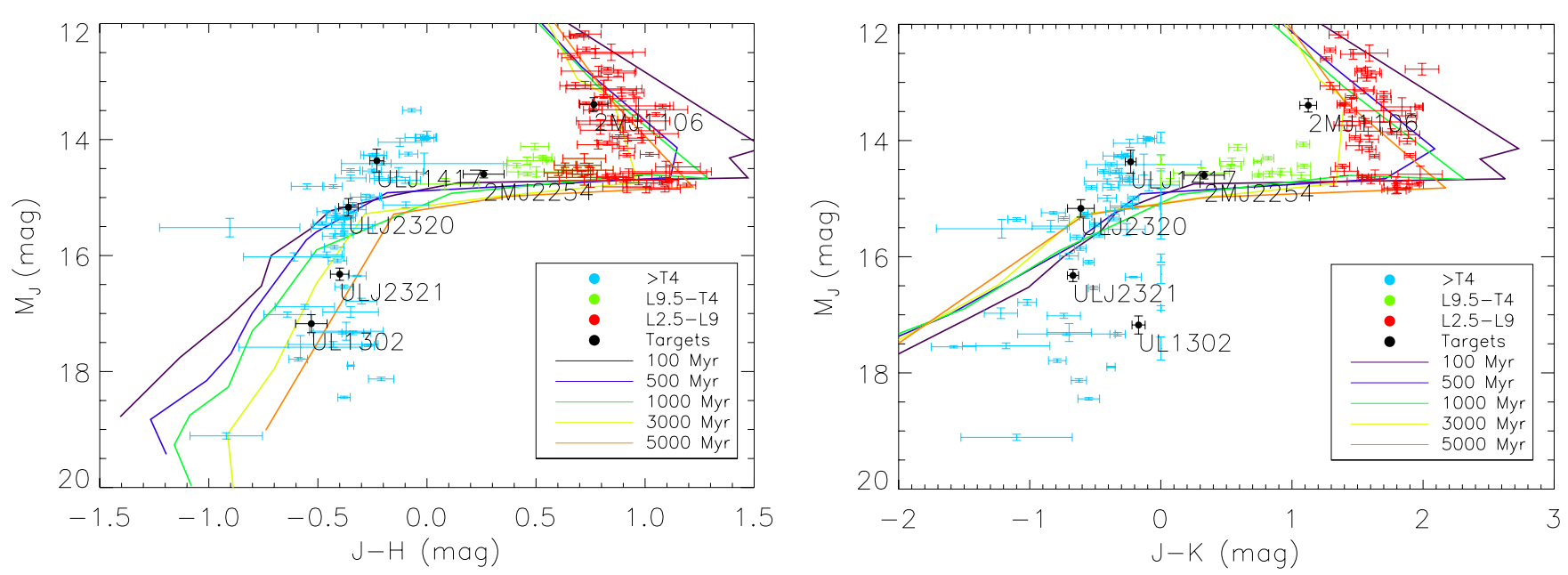

Fig. 1. Color-magnitude diagram in the MKO system showing the brown dwarf sample from Dupuy \& Liu (2012) (except M-type brown dwarfs), our targets and the BT Settl-models (Allard et al. 2012). The objects in the Dupuy \& Liu (2012) sample with spectral type between L2.5-L9 are shown in red; the objects with spectral types between L9.5 and T4 are shown in green; and the objects with spectral type $>$ T4 are shown in light blue. Our targets, which are listed in Table 1, are shown in black. We overplot the evolutionary models from Allard et al. (2012) in MKO photometry for different ages from $100 \mathrm{Myr}$ to $5000 \mathrm{Myr}$.

- Atmospheric image motions. Atmospheric effects also limit the precision of the astrometry in several different ways, such as intensity scintillation, image blurring, image motion and speckle structure. We estimated this effect using the expression given in Lindegren (1980): $\epsilon^{2} \simeq 0.71 R^{2 / 3} T^{-1}$ under the condition: $14 T \gg 4300 R \gg d$. The parameter $T$ is the integration time in seconds ( $60 \mathrm{~s} \times 15$ single images), $R$ is the diameter of the field in radians $\left(4.5 \times 10^{-3} \mathrm{rad}\right)$ and $d$ is the diameter of the telescope in meters $(3.5 \mathrm{~m})$. The variance for the atmospheric image motion is estimated to be 5 mas per epoch.

- DCR effect. This error was calculated as explained in Sect. 3.2, and it is typically 1 mas per epoch.

- Plate solution. Distortions are introduced by the instrument because it is not sufficiently stable, as it is dismounted between two epochs. The program SCAMP calculates the best astrometric solution performing a $\chi^{2}$ minimization and calculates the distortions using the relative positions of the reference objects between the reference catalog and the catalogs of all epochs. The value of the residual distortion is between 5 and 10 mas for bright stars per epoch. The distortions due to the images stacking are negligible. We measure up to $0.04 \%$ distortions or 0.5 pixel distortions from center to corner (Bailer-Jones et al. 2000).

- Error in the conversion from relative to absolute parallaxes. It is calculated Sect. 3.3.2. The typical value of these uncertainties is $\sim 1.5$ mas.

\section{Results}

The astrometric and photometric results for the six objects are compiled in Table 2. Column 1 contains the object's name. Columns 2 and 3 give the derived absolute proper motions in RA $\left(\mu_{\alpha}\right)$ and Dec $\left(\mu_{\delta}\right)$ for the targets; Col. 4 gives the relative parallax $\left(\pi_{\text {rel }}\right)$. In Col. 5 , we provide the absolute parallax $\left(\pi_{\mathrm{abs}}\right)$. Column 6 contains the derived distance $(d)$ from the absolute parallax results. Column 7 provides the photometric distance $\left(d_{\text {phot }}\right)$ in pc, using the relation published in Goldman et al. (2010), and Col. 8 contains the values for $\chi^{2}$ and $N_{\text {d.o.f. }}$, which is the number of degrees of freedom.

To characterize our targets, we plot them in a colormagnitude diagram (CMD) with the $177 \mathrm{~L}, \mathrm{~L}-\mathrm{T}$, and T brown dwarfs published by Dupuy \& Liu (2012) with magnitudes in the MKO system. Our magnitudes were originally in the 2MASS photometric system, so that we use the relation published in Stephens \& Leggett (2004) to transform between the 2MASS photometric system and the MKO system. The rest of the targets have UKIDSS photometry, which is similar to the MKO photometry (Hewett et al. 2006). For the target ULAS 2320+1448 we provide the photometry from Murray et al. (2011), also similar to MKO. We plot $\left(J-H, M_{J}\right)$ and $\left(J-K, M_{J}\right)$ and $(W 1-W 2$, $\left.M_{w} 1\right)$ using WISE photometry.

We overplot our CMD with the BT-Settl models by Allard et al. (2012). The CMDs are shown in Fig. 1 with overplotted 


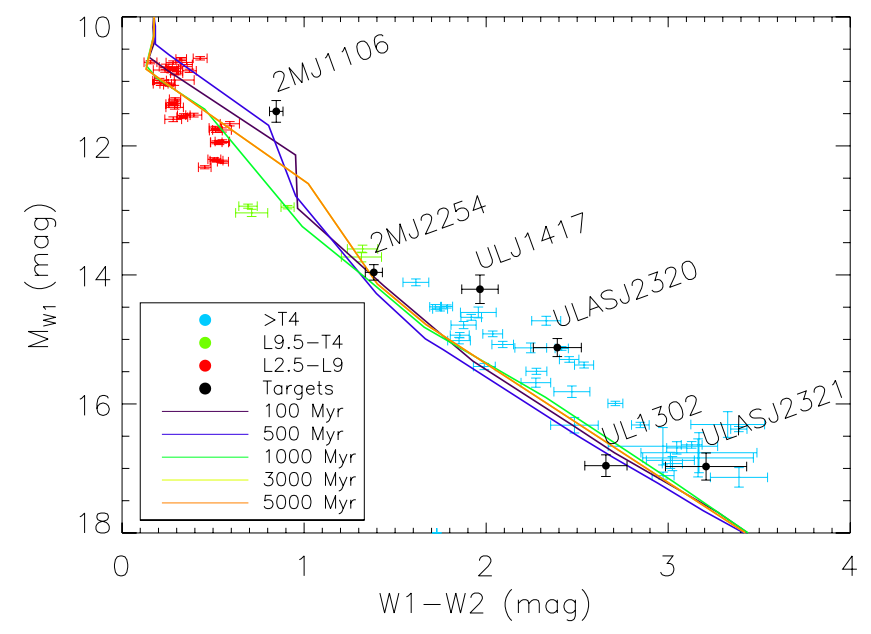

Fig. 2. Color-magnitude diagram in WISE photometry with W1-W2 color vs. $M_{W 1}$ (WISE). We plot the objects from Dupuy \& Liu (2012) with WISE photometry. L2.5-L9 brown dwarfs are colored in red; objects with spectral types between L9.5 and T4 are green; and objects with spectral type $>\mathrm{T} 4$ are colored in light blue. Our targets are plotted in black. We overplot the evolutionary models from Allard et al. (2012) in WISE photometry for different ages, from $100 \mathrm{Myr}$ to $5000 \mathrm{Myr}$.

isochrones from Allard et al. (2012). We show the stellar paths for all the objects in the appendix.

\section{Discussion}

\subsection{MASS J11061197+2754225}

This object was discovered by Looper et al. (2007). It was identified using a near-IR proper-motion survey based on multiepoch data from the Two Micron All Sky Survey (2MASS). It was classified as a T2.5 BD using the IRTF SpeX spectrograph in low-resolution mode with a resolution of $R \sim 150$. Looper et al. (2007) estimated the spectrophotometric distance as $15.5 \pm 1.2 \mathrm{pc}$, which was calculated using the spectral types that were derived in the same article and by using the Liu et al. (2006) spectral type versus magnitude relation without known binaries. Burgasser et al. (2010) proposed this target to be a strong binary candidate in a T0.0 \pm 0.2 and $T 4.5 \pm 0.2$ system, although Looper et al. (2008) performed high angular resolution imaging with NIRC2/Keck II, finding only one source.

The location of the object in Figs. 1 and 2 seem to give a luminosity inconsistent with the classification provided before (Looper et al. 2007; Kirkpatrick et al. 2010) by using indices in the near-IR. Looper et al. (2007) mentions that J1106+27 is distinctly $(>1-\sigma)$ bluer in the $z-J$ band in SDSS than all the T2 brown dwarfs in their sample. In our CMD (see Figs. 1 and 2), the object is also overluminous by $\sim 1 \mathrm{mag}$. Due to its overluminosity, this object is a good candidate for a binary system or even high order multiplicity, although this issue should be studied in more detail using high resolution images or high resolution spectroscopy. The spectroscopic distance provided by Looper et al. (2007) is not compatible with the distance derived from the trigonometric absolute parallax within the error bars. The calculated distance is $20.6_{-1.2}^{+1.0} \mathrm{pc}$.

\subsection{ULAS J130217.21+130851.2}

This object was discovered by Burningham et al. (2010), and it was classified as a T8.5 BD by using NIRI on the Gemini North Telescope and IRCS on the Subaru telescope on Mauna Kea, Hawaii. The spectral classification was based on the spectral indices given by Burgasser et al. (2006b). The object ULAS J1302+13 was later re-typed as T8 by Cushing et al. (2011).

With regard to Figs. 1 and 2, this object is not overluminous. The value of the distance has been calculated for the first time and is $15.4_{-1.4}^{+1.1} \mathrm{pc}$.

\subsection{ULAS J141756.22+133045.8}

The discovery of ULAS J141756.22+133045.8 was first published by Scholz (2010). It was classified as T5.5 $\pm 1.0 \mathrm{BD}$ and based on colors and absolute magnitudes from UKIDSS and SDSS. The absolute proper motions were also estimated, using UKIDSS and SDSS data with a baseline of five years, but only with three different epochs.

The result supplied in Scholz (2010) for the absolute proper motions were $\mu_{\alpha} \cos \delta=-76 \pm 3$ mas $/ \mathrm{yr}$ and $\mu_{\delta}=77 \pm 3 \mathrm{mas} / \mathrm{yr}$. Scholz (2010) pointed out that the formal errors were very small and were due to the few number of epochs and so that the errors could be unrealistic. The estimated errors by Scholz (2010) were $24 \mathrm{mas} / \mathrm{yr}$, which was the scatter of their data around the best fit to the model.

Burningham et al. (2013) presented YJHK spectroscopy for this object using the Gemini Near Infrared Spectrograph (GNIRS) and derived a type a spectral type of $T 5 \pm 0.5$.

Comparing the results from Scholz (2010) for the proper motions with our results and taking the errors into account, the results are compatible. With regard to Fig. 2, we can conclude that this object is slightly overluminous in WISE by around 0.2 mag. In Fig. 1, this overluminosity is not that clear, although the object is at the edge of the bulk of objects. Our result for the distance is $30.3_{-3.8}^{+2.5} \mathrm{pc}$.

\subsection{MASS J22541892+3123498}

The object 2MASS J22541892+3123498 was first published as a brown dwarf by Burgasser et al. (2002a). It was classified as a T5V BD by using spectral observations in NIR with $R \sim 1200$ and by calculating the spectral indices, as explained in Burgasser et al. (2002a). Afterward, the spectral type was recalculated using the criteria given in Burgasser et al. (2003) as a standard T4 BD (Burgasser et al. 2004, 2006b). The proper motions were also estimated by Jameson et al. (2008) using WFCAM at UKIRT from February to August 2006. Their result for the proper motions was $\mu_{\alpha} \cos \delta=68 \pm 15 \mathrm{mas} / \mathrm{yr}$ and $\mu_{\delta}=200 \pm 11 \mathrm{mas} / \mathrm{yr}$.

Our results for the proper motions agree with the result given by Jameson et al. (2008) within the error bars. This object is not overluminous. The provided distance is $13.9_{-0.6}^{+0.5} \mathrm{pc}$, and it has been calculated for the first time.

\subsection{ULAS J232035.28+144829.8}

The discovery of this object was first published by Burningham et al. (2010), and it was classified as a T5 spectral type using medium resolution spectroscopy. In Scholz (2010), the object is classified as T7.0 \pm 2.0 using colors from UKIDSS and SDSS. In Murray et al. (2011), the spectral indices were recalculated, using data from NIRI on the Gemini-North telescope with a spectral resolution of $R \sim 460$; the new classification is T6 \pm 1 .

In Scholz (2010), the proper motions were given as $\mu_{\alpha} \cos \delta=387 \pm 5 \mathrm{mas} / \mathrm{yr}$ and $\mu_{\delta}=121 \pm 2 \mathrm{mas} / \mathrm{yr}$. These proper motions were calculated with a baseline of seven years; nevertheless, there were only three epochs in total, and two of 
them very close to each other. The estimated error in Scholz (2010) due to the scatter around the best fit to the model was $11 \mathrm{mas} / \mathrm{yr}$.

In Murray et al. (2011), the proper motions and the distance were also estimated performing a photometric follow-up using UKIDSS, by obtaining proper motions of $\mu_{\alpha} \cos \delta=399 \pm$ $26 \mathrm{mas} / \mathrm{yr}, \mu_{\delta}=122 \pm 26 \mathrm{mas} / \mathrm{yr}$, and distance of $24 \pm 5 \mathrm{pc}$.

The proper motions, which we obtained, agree to the values provided by Scholz (2010) and Murray et al. (2011) within the error bars, but our values are more precise. We also agree with Murray et al. (2011) on the distance to within the error bars. This object is not overluminous (see Figs. 1 and 2). The calculated distance is $21.1_{-2.2}^{+1.6} \mathrm{pc}$.

\subsection{ULAS J232123.79+135454.9}

The brown dwarf discovery of ULAS J232123.79+135454.9 was first published by Scholz (2010). It was classified with a spectral type of T7.5 \pm 1.5 using UKIDSS and SDSS colors. This object was also classified in Burningham et al. (2010) as a T7.5 using medium resolution spectroscopy.

Scholz (2010) also provided the proper motions using SDSS and UKIDSS with a baseline of seven years and four epochs taken along this baseline. The proper motions were given as $\mu_{\alpha} \cos \delta=56 \pm 15 \mathrm{mas} / \mathrm{yr}$ and $\mu_{\delta}=-577 \pm 10 \mathrm{mas} / \mathrm{yr}$ with an estimated error of $10 \mathrm{mas} / \mathrm{yr}$ due to the scatter of the data around the fit to the best model. Kirkpatrick et al. (2012) provided a distance limit of approximately $20 \mathrm{pc}$.

The object ULAS J232123.79+135454.9 is not overluminous (see Figs. 1 and 2). We agree with Scholz (2010) on the result for the proper motion within the error bars. Kirkpatrick et al. (2012) adopted a distance of $14.1 \mathrm{pc}$. Our result for the distance is $11.8_{-0.6}^{+0.5} \mathrm{pc}$.

\section{Conclusions}

We have measured the trigonometric parallaxes of six $\mathrm{T}$ brown dwarfs for the first time with spectral types between T2.5 and T7.5.

We compare our results to the spectrophotometric distance given by Goldman et al. (2010) and to the results of the spectrophotometric distance and proper motions provided in other studies such as Scholz (2010), Looper et al. (2007) and Kirkpatrick et al. (2012) among others. Our results generally agree well with other studies, but they are more precise.

We also compare the locations of our targets in $\left(J-H, M_{J}\right)$ and $\left(J-K, M_{J}\right)$ CMDs to those of the objects by Dupuy \& Liu (2012) and to the evolutionary tracks by Allard et al. (2012).

Four of our six targets are not overluminous. Nevertheless, the object ULAS J141756.22+133045.8 is slightly overluminous in the WISE CMD. It has an absolute magnitude in W1, which is around $0.2 \mathrm{mag}$ brighter than the objects with the same spectral types. Its overluminosity is not seen in the CMDs in MKO photometry. The object 2MASS J11061197+2754225 is more than 1 mag overluminous in all the bands, pointing to binarity or even higher multiplicity. To confirm these results, high resolution imaging and high resolution spectroscopy is needed.

Acknowledgements. We acknowledge J. A. Caballero, M. R. Zapatero Osorio, V. J. S. Béjar, W. Brandner, A. Bayo, A. Burgasser and B. Burningham for their help, their corrections and contructive criticism. We thank in particular our referee T. Dupuy for his useful advice, which substantially helped to improve our paper. This work was supported by Sonderforschungsbereich SFB 881 The Milky Way System (subproject B6) of the German Research Foundation (DFG).

\section{References}

Allard, F., Homeier, D., \& Freytag, B. 2012, in IAU Symp., 282, eds. M. T. Richards, \& I. Hubeny, 235

Bailer-Jones, C. A., Bizenberger, P., \& Storz, C. 2000, in SPIE Conf. Ser., 4008, eds. M. Iye, \& A. F. Moorwood, 1305

Basri, G., Marcy, G. W., \& Graham, J. R. 1995, BAAS, 27, 1214

Becklin, E. E., \& Zuckerman, B. 1988, Nature, 336, 656

Bertin, E. 2006, in Astronomical Data Analysis Software and Systems XV, eds.

C. Gabriel, C. Arviset, D. Ponz, \& S. Enrique, ASP Conf. Ser., 351, 112

Bertin, E., \& Arnouts, S. 1996, A\&AS, 117, 393

Burgasser, A. J., Kirkpatrick, J. D., Brown, M. E., et al. 2002a, ApJ, 564, 421

Burgasser, A. J., Marley, M. S., Ackerman, A. S., et al. 2002b, ApJ, 571, L151

Burgasser, A. J., Geballe, T. R., Golimowski, D. A., et al. 2003, in Brown Dwarfs, ed. E. Martín, IAU Symp. (San Francisco: ASP), 211, 377

Burgasser, A. J., McElwain, M. W., Kirkpatrick, J. D., et al. 2004, AJ, 127, 2856 Burgasser, A. J., Burrows, A., \& Kirkpatrick, J. D. 2006a, ApJ, 639, 1095

Burgasser, A. J., Geballe, T. R., Leggett, S. K., Kirkpatrick, J. D., \& Golimowski, D. A. 2006b, ApJ, 637, 1067

Burgasser, A. J., Vrba, F. J., Lépine, S., et al. 2008, ApJ, 672, 1159

Burgasser, A. J., Cruz, K. L., Cushing, M., et al. 2010, ApJ, 710, 1142

Burningham, B., Pinfield, D. J., Lucas, P. W., et al. 2010, MNRAS, 406, 1885

Burningham, B., Cardoso, C. V., Smith, L., et al. 2013, MNRAS, 433, 457

Burrows, A., Sudarsky, D., \& Hubeny, I. 2006, ApJ, 640, 1063

Cushing, M. C., Kirkpatrick, J. D., Gelino, C. R., et al. 2011, ApJ, 743, 50

Dahn, C. C., Harris, H. C., Vrba, F. J., et al. 2002, AJ, 124, 1170

Dupuy, T. J., \& Liu, M. C. 2012, ApJS, 201, 19

Faherty, J. K., Burgasser, A. J., Walter, F. M., et al. 2012, ApJ, 752, 56

Fruchter, A. S., \& Hook, R. N. 2002, PASP, 114, 144

Goldman, B., Marsat, S., Henning, T., Clemens, C., \& Greiner, J. 2010, MNRAS, 405, 1140

Hewett, P. C., Warren, S. J., Leggett, S. K., \& Hodgkin, S. T. 2006, MNRAS, 367,454

Hog, E., Fabricius, C., Makarov, V. V., et al. 2000, VizieR Online Data Catalog, $\mathrm{I} / 259$

Jameson, R. F., Casewell, S. L., Bannister, N. P., et al. 2008, MNRAS, 384, 1399 Kaczmarczik, M. C., Richards, G. T., Mehta, S. S., \& Schlegel, D. J. 2009, AJ, 138,19

Kirkpatrick, J. D., Looper, D. L., Burgasser, A. J., et al. 2010, ApJS, 190, 100

Kirkpatrick, J. D., Gelino, C. R., Cushing, M. C., et al. 2012, ApJ, 753, 156

Lang, D., Hogg, D. W., Mierle, K., Blanton, M., \& Roweis, S. 2010, AJ, 139, 1782

Lindegren, L. 1980, A\&A, 89, 41

Liu, M. C., Leggett, S. K., Golimowski, D. A., et al. 2006, ApJ, 647, 1393

Liu, M. C., Leggett, S. K., \& Chiu, K. 2007, ApJ, 660, 1507

Looper, D. L., Kirkpatrick, J. D., \& Burgasser, A. J. 2007, AJ, 134, 1162

Looper, D. L., Gelino, C. R., Burgasser, A. J., \& Kirkpatrick, J. D. 2008, ApJ, 685, 1183

Marocco, F., Smart, R. L., Jones, H. R. A., et al. 2010, A\&A, 524, A38

Monet, D. G., Dahn, C. C., Vrba, F. J., et al. 1992, AJ, 103, 638

Monet, D. G., Levine, S. E., Canzian, B., et al. 2003, AJ, 125, 984

Murray, D. N., Burningham, B., Jones, H. R. A., et al. 2011, MNRAS, 414, 575

Mutchler, M., \& Fruchter, A. 1997, BAAS, 29, 1271

Nakajima, T., Oppenheimer, B. R., Kulkarni, S. R., et al. 1995, Nature, 378, 463

Perryman, M. A. C. 1997, BAAS, 29, 1314

Pravdo, S. H., \& Shaklan, S. B. 1996, ApJ, 465, 264

Rebolo, R., Zapatero Osorio, M. R., \& Martín, E. L. 1995, Nature, 377, 129

Saumon, D., \& Marley, M. S. 2008, ApJ, 689, 1327

Schilbach, E., Röser, S., \& Scholz, R.-D. 2009, A\&A, 493, L27

Scholz, R.-D. 2010, A\&A, 515, A92

Stephens, D. C., \& Leggett, S. K. 2004, PASP, 116, 9

Stone, R. C. 1996, PASP, 108, 1051

Tinney, C. G., Burgasser, A. J., \& Kirkpatrick, J. D. 2003, AJ, 126, 975

Tsuji, T., Ohnaka, K., Aoki, W., \& Nakajima, T. 1996, A\&A, 308, L29

Vrba, F. J., Henden, A. A., Luginbuhl, C. B., et al. 2004, AJ, 127, 2948

Pages 7 to 8 are available in the electronic edition of the journal at http://www. aanda. org 
E. Manjavacas et al.: Parallax measurements of cool brown dwarfs

\section{Appendix A: Stellar paths of the targets}

We include the stellar paths, which represent the change in right ascension (RA) and declination (Dec) in time for our targets in this appendix.
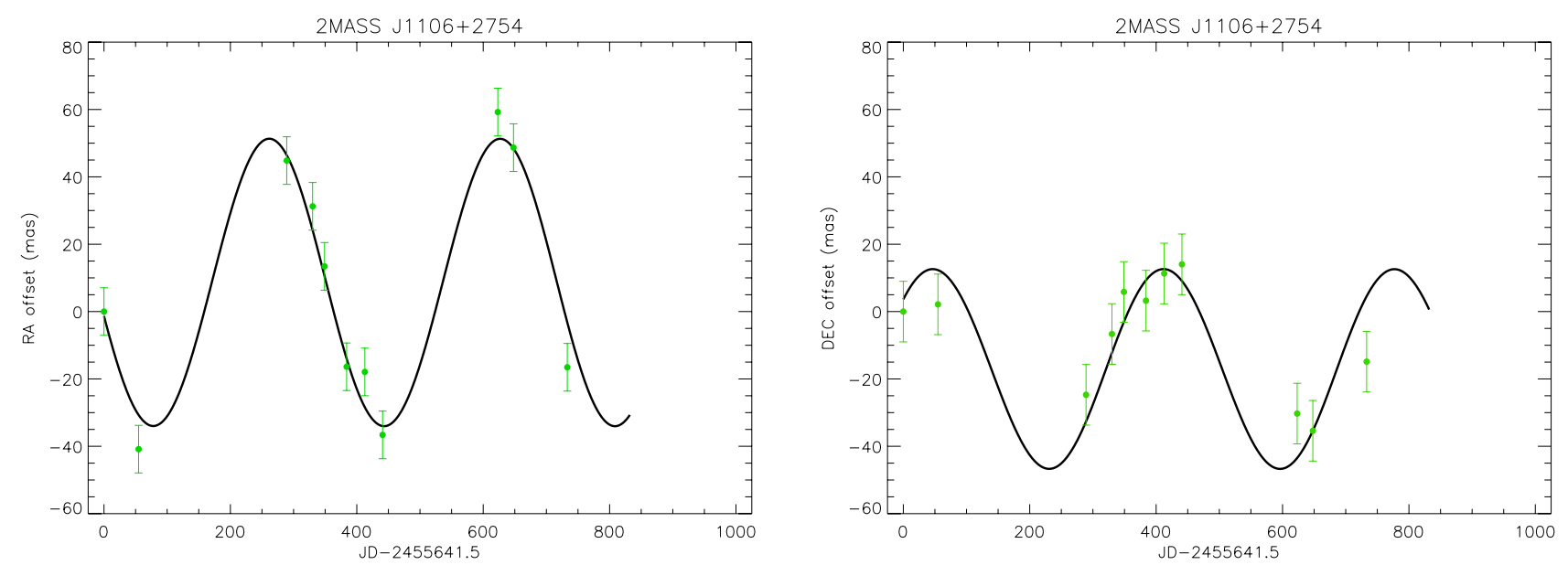

Fig. A.1. Stellar paths for the object 2MASS J11061197+2754225.
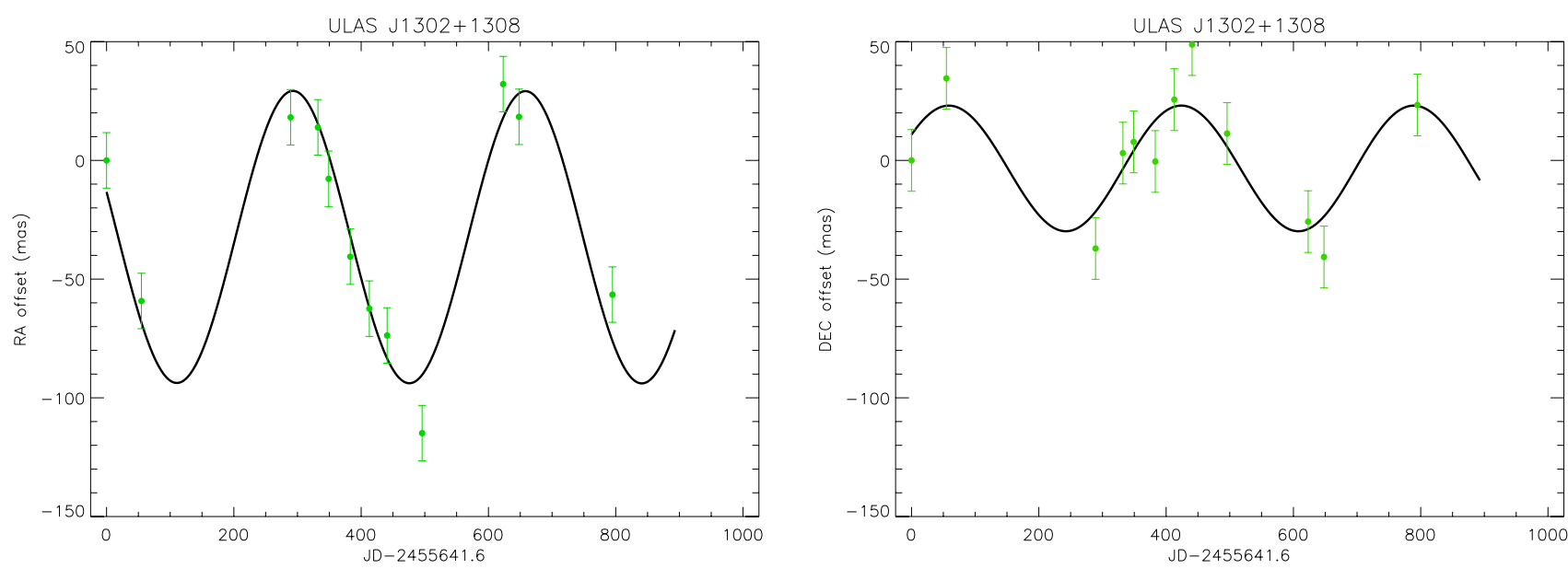

Fig. A.2. Stellar paths for the object ULAS J130217.2+130851.2.
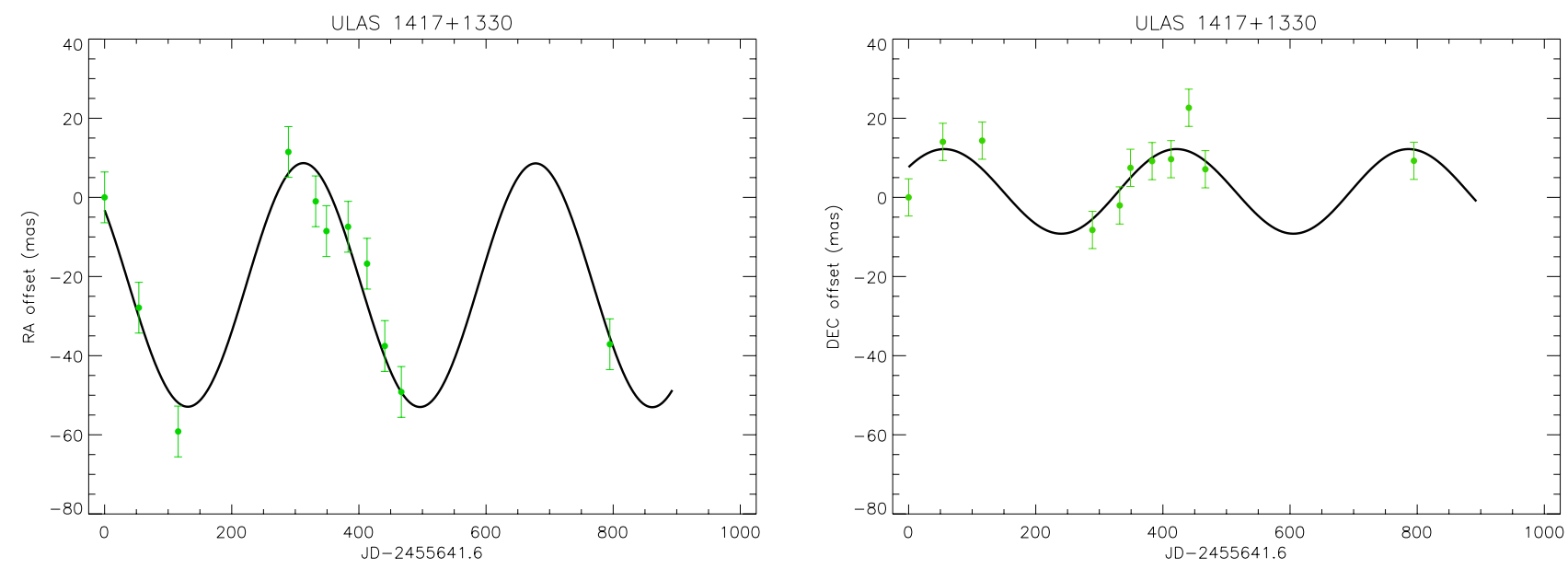

Fig. A.3. Stellar paths for the object ULAS J141756.22+133045.8. 
A\&A 560, A52 (2013)
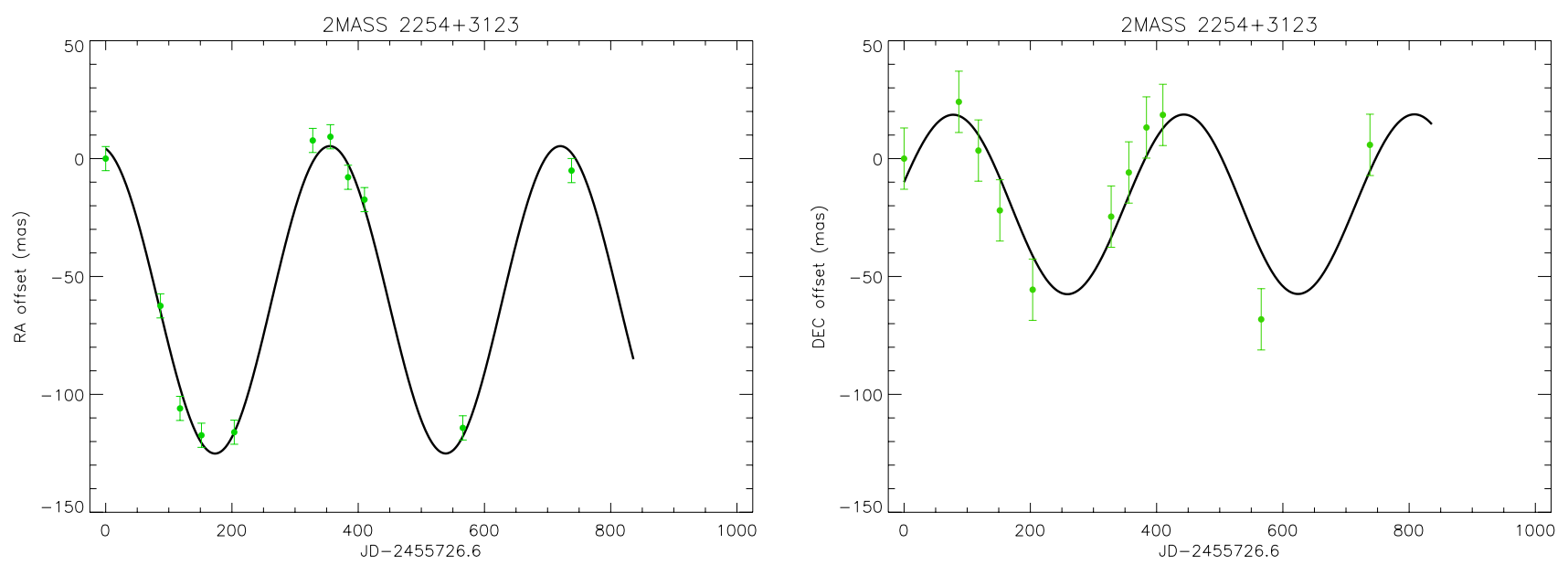

Fig. A.4. Stellar paths for the object 2MASS J22541892+3123498.
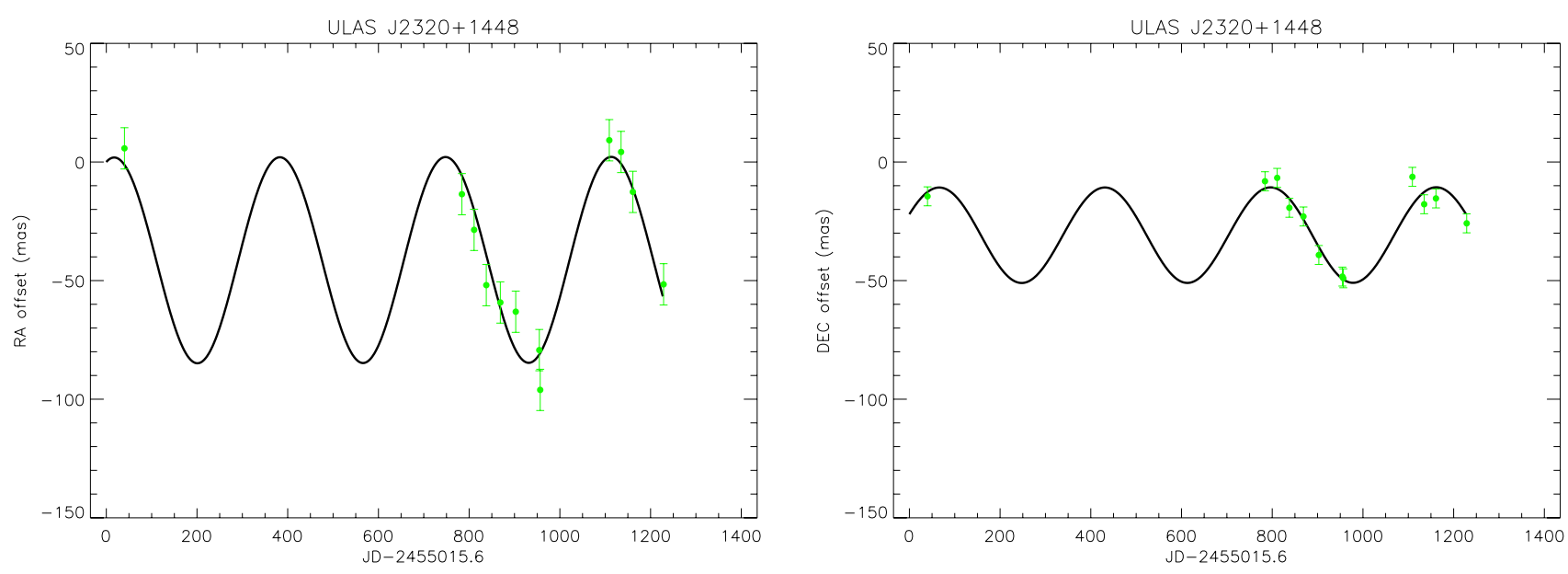

Fig. A.5. Stellar paths for the object ULAS J232035.28+144829.8. The first epoch is an archival Omega-2000 observation.
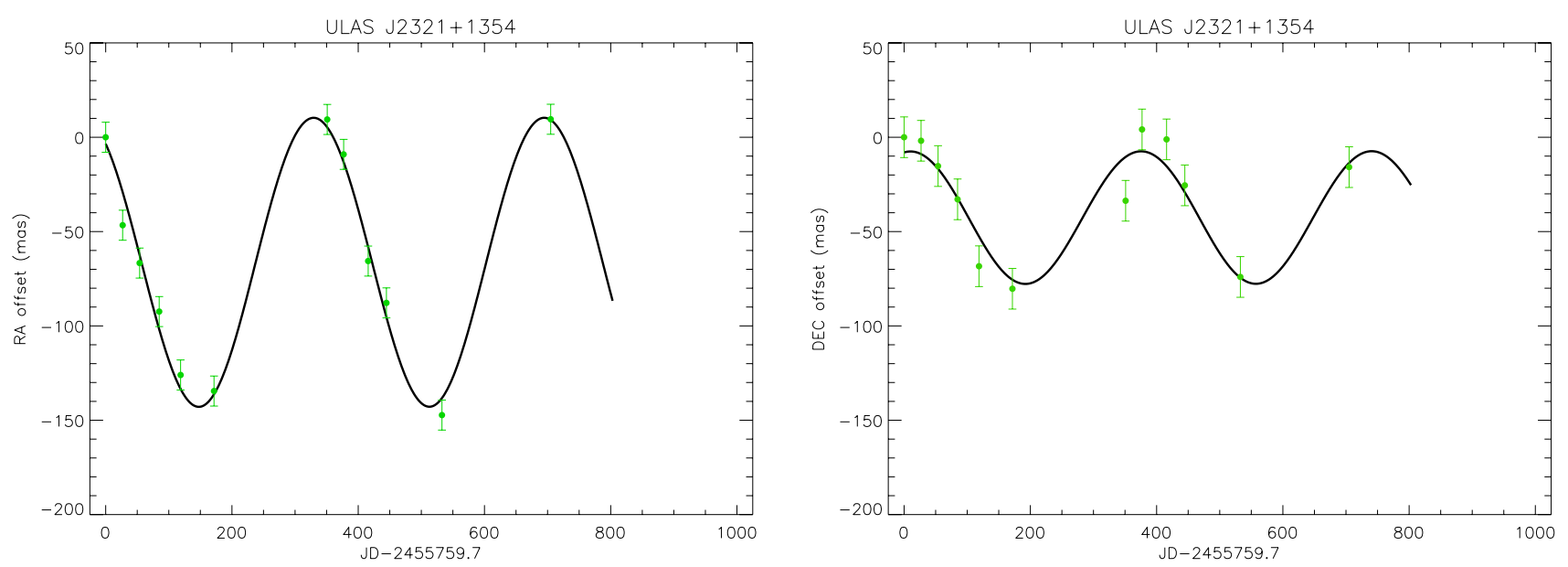

Fig. A.6. Stellar paths for the object ULAS J232123.7+1354454. 